\title{
Will Residual Antibiotics in Hen Eggs Elicit Health Risks by Impairing Human Intestinal Microbiome?
}

\author{
Yu-Hua Zhang\#, Jing Bai", Chong-Feng Li, Cheng-Rui Zhao, Yan-Wei Liu*, Jing-Zhang Wang* and Rui- \\ Ying Zhang* \\ College of Life Sciences and Food Engineering, College of Medicine, Affiliated Hospital, Hebei University of Engineering, PR China \\ ${ }^{\#}$ These authors contributed equally to this study and should be co-first authors
}

*Corresponding author: Yan-wei, College of Life Sciences and Food Engineering, College of Medicine, Affiliated Hospital, Hebei University of Engineering, Handan 056038, PR China

Jing-Zhang, College of Life Sciences and Food Engineering, College of Medicine, Affiliated Hospital, Hebei University of Engineering, Handan 056038, PR China

Rui-Ying, College of Life Sciences and Food Engineering, College of Medicine, Affiliated Hospital, Hebei University of Engineering, Handan 056038, PR China

\section{ARTICLE INFO}

Received: 蔧 July 13, 2019

Published: 慧 July 26, 2019

Citation: Yu-Hua Zhang, Jing Bai, ChongFeng Li, Cheng-Rui Zhao, Yan-Wei Liu, Jing-Zhang Wang, Rui-Ying Zhang. Will Residual Antibiotics in Hen Eggs Elicit Health Risks by Impairing Human Intestinal Microbiome?. Biomed J Sci \& Tech Res 20(1)2019. BJSTR. MS.ID.003396.

\begin{abstract}
Various residual antibiotics have been detected in hen eggs and pose a health threat worldwide, especially in Developing Countries and Underdeveloped Countries. The opinion presented here asserts that infinitesimal antibiotics from dietary eggs may accumulate in the human intestines and gradually disturb the intestinal microbiome and further dysbiosis of intestinal microbiome may be a potential risk to human health via impairing the functions of multiple internal organs in human beings.
\end{abstract}

Keywords: Intestinal microbiome; Hen egg; Antibiotics; Microbial dysbiosis; Human disease

\section{Introduction}

According to recent studies, food-borne microorganisms are closely related to human diseases, and dysbiosis of the intestinal microbiome was found to be a common pathogenic mechanism underlying several kinds of human diseases such as cancer, cardiovascular disease, inflammatory bowel disease, neural deficiency, etc. [1-6]. Hen eggs provide people with abundant nutrients but may raise some health risks. For example, egg proteins may induce allergic responses, which is to some extent related to insufficient digestive function in the human intestine [7]. For another example, choline-class substrates in eggs can be decomposed by human intestinal microorganisms and then produce trimethylamine $\mathrm{N}$-oxide (TMAO) which increases the risks of atherosclerosis and cardiovascular disorders $[8,9]$. These findings hint that intestinal microorganisms play a key role in mediating potential health risks of egg-derived molecules in human beings.
Discussions on Hen Eggs, Residual Antibiotics, Intestinal Microbiome and Human Diseases

\section{Residual Antibiotics in Eggs Lead to Serious Dietary Problems}

Recently, we noticed that residual antibiotics in hen eggs may pose numerous health risks. In order to improve hen survival and egg production rate, excessive addition of antibiotics into animal feed is very prevalent worldwide, especially in Developing and Undeveloped Countries and those areas with weak supervisory measures. As a result, a variety of antibiotics have been detected in hen eggs and egg-products, including oxytetracycline hydrochloride, chloramphenicol, marbofloxacin, difloxacin, sarafloxacin, enrofloxacin, etc. [10-15]. Although the concentrations of these antibiotics in eggs may be low, the potential harm of their long-term accumulation should not be underestimated. 
Antibiotics in Egg May Gradually Disrupt Microbial Composition in Intestines

In fact, hen egg is a kind of regular and popular food in many regions of the world. Theoretically, once residual antibiotics in eggs inhibit intestinal microorganisms for a long time, they may gradually disrupt the bacterial compositions and species of human intestinal microbiome and even some beneficial metabolic products of intestinal microorganisms, which consequently results in dysbiosis of human intestinal microbiome and lays a pathogenic foundation for relevant human diseases.

\section{Dysbiosis of Intestinal Microorganisms May Induce Various Human Diseases}

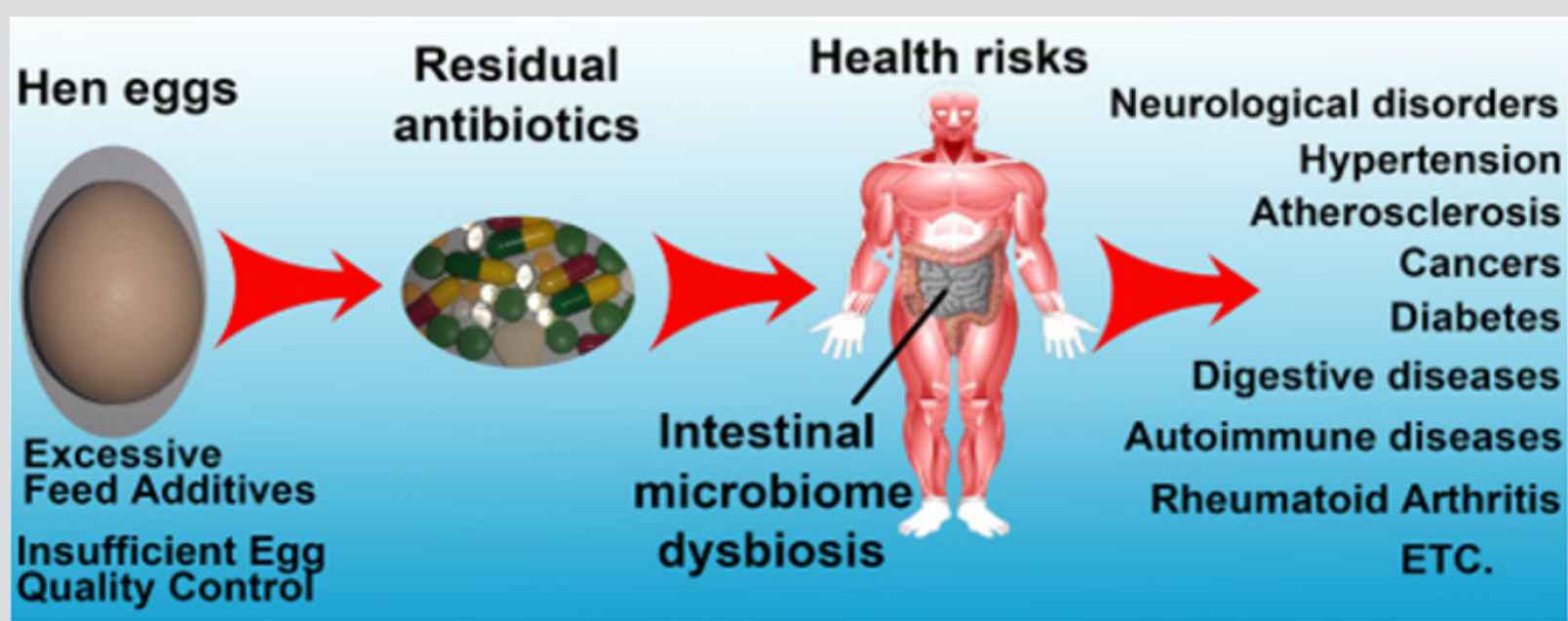

Figure 1: Residual antibiotics from hen eggs may impair the balance of intestinal microbiome and increase health risks in human beings.

There is a symbiotic relationship between beneficial intestinal microbiome and human beings, some substances produced by intestinal microorganisms have important effects on human health. Increasing evidence suggests that intestinal microbial dysfunction can cause a number of human diseases, possibly including but not limited to cancers, cardiovascular diseases, neurological disorders, diabetes, autoimmune problems, etc., as shown in Figure 1 [13,16-19]. Frankly, eggs may only contain a low concentration of residual antibiotics, and their potential suppression on intestinal microorganisms may not be obvious enough to cause disease in a short time. However, long-term health risks of residual antibiotics should not be neglected and deserves more safety concern and more medical attention.

\section{Conclusion}

Taken together, residual antibiotics in hen eggs may inhibit and disrupt human intestinal microbiome gradually leading to multiple health risks. This is a new and interesting medical prediction putting forward a pathogenic link between dietary antibiotics, intestinal microorganisms and future high-incidence human diseases. The opinion underlines the long-term health risks potentially induced by dietary antibiotics especially in some areas without strict limitation to antibiotic usage. Hence, it lays a novel foundation for diet- based pathogenesis of human diseases and calls for strictly controlling antibiotic additives in chicken breeding as well as strengthening detection of antibiotics in egg production, with the aim of reducing health risks to human beings in the future.

\section{Conflict of Interest Statement}

None.

\section{Acknowledgement}

This work was supported by the Hebei Provincial Social Science Fund Projects (No. HB19SH020; No. 2019030401038) and Hebei Provincial Science and Technology Project (No. 182777107D).

\section{References}

1. Zhuang H, Cheng L, Wang Y, Zhang YK, Zhao MF, et al. (2019) Dysbiosis of the gut microbiome in lung cancer. Front Cell Infect Mi 9: 112.

2. de la Cuesta Zuluaga J, Mueller NT, Alvarez Quintero R, Velasquez Mejia EP, Sierra JA, et al. (2019) Higher fecal short-chain fatty acid levels are associated with gut microbiome dysbiosis, obesity, hypertension and cardiometabolic disease risk factors. Nutrients 11(1): E51.

3. Wang JZ, Du WT, Xu YL, Cheng SZ, Liu ZJ (2017) Gut microbiome-based medical methodologies for early-stage disease prevention. Microb Pathogenesis 105: 122-130.

4. Messens W, Hempen M, Koutsoumanis K (2018) Use of predictive modelling in recent work of the panel on biological hazards of the european food safety authority. Microbial Risk Analysis 10: 37-43.

5. Ebel ED, Williams MS, Ward Gokhale LA, Kisselburgh HM (2019) Assessing the maximum size of annual foodborne outbreaks in the united states: An analysis of 1973-2016 outbreaks. Microbial Risk Analysis.

6. Caffrey N, Invik J, Waldner CL, Ramsay D, Checkley SL (2019) Risk assessments evaluating foodborne antimicrobial resistance in humans: A scoping review. Microbial Risk Analysis 11: 31-46.

7. Yokooji T, Hamura K, Matsuo H (2013) Intestinal absorption of lysozyme, an egg-white allergen, in rats: Kinetics and effect of nsaids. Biochem Bioph Res Co 438(1): 61-65. 
8. Lemos BS, Medina Vera I, Malysheva OV, Caudill MA, Fernandez ML (2018) Effects of egg consumption and choline supplementation on plasma choline and trimethylamine-n-oxide in a young population. J Am Coll Nutr 15:1-8.

9. Ding L, Chang M, Guo Y, Zhang L, Xue C, et al. (2018) Trimethylaminen-oxide (TMAO)-induced atherosclerosis is associated with bile acid metabolism. Lipids Health Dis 17(1): 286.

10. Long XY, Gong JN, Deng M, Wu D, Chen Z, et al. (2019) Determination of oxytetracycline hydrochloride in milk and egg white samples using ru(bipy) ${ }_{3}{ }^{2+}$-ce $\left(\mathrm{so}_{4}\right)_{2}$ chemiluminescence. Luminescence 34(3): 316-323.

11. Peris Vicente J, Iborra Millet JJ, Albiol Chiva J, Carda Broch S, Esteve Romero J (2019) A rapid and reliable assay to determine flumequine, marbofloxacin, difloxacin, and sarafloxacin in commonly consumed meat by micellar liquid chromatography. J Sci Food Agr 99(3): 1375-1383.

12. Teglia CM, Gonzalo L, Culzoni MJ, Goicoechea HC (2019) Determination of six veterinary pharmaceuticals in egg by liquid chromatography: Chemometric optimization of a novel air assisted-dispersive liquidliquid microextraction by solid floating organic drop. Food Chem 273 194-202.

13. Wu Q, Peng DP, Liu QY, Shabbir MA, Sajid A, et al. (2019) A novel microbiological method in microtiter plates for screening seven kinds of widely used antibiotics residues in milk, chicken egg and honey. Front Microbiol 10: 436

\section{ISSN: 2574-1241}

DOI: 10.26717/BJSTR.2019.20.003396

YW Liu, JZ Wang, RY Zhang. Biomed J Sci \& Tech Res

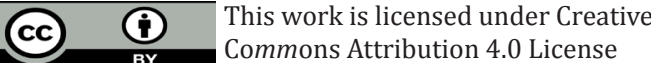

Submission Link: https://biomedres.us/submit-manuscript.php
14. Xu X, Xu XY, Han M, Qiu ST, Ho X (2019) Development of a modified quechers method based on magnetic multiwalled carbon nanotubes for the simultaneous determination of veterinary drugs, pesticides and mycotoxins in eggs by uplc-ms/ms. Food Chem 276: 419-426.

15. Zhang XN, Song Y, Jia Q, Zhang L, Zhang W, et al. (2019) Simultaneous determination of 58 pesticides and relevant metabolites in eggs with a multi-functional filter by ultra-high-performance liquid chromatography-tandem mass spectrometry. J Chromatogr A 1593: 8190.

16. Xia GH, You C, Gao XX, Zeng XL, Zhu JJ, et al. (2019) Stroke dysbiosis index (SDI) in gut microbiome are associated with brain injury and prognosis of stroke. Front Neurol 10: 397.

17. Wang QQ Xu R (2019) Data-driven multiple-level analysis of gutmicrobiome-immune-joint interactions in rheumatoid arthritis. Bmc Genomics 20: 124.

18. Katsi V, Didagelos M, Skevofilax S, Armenis I, Kartalis A, et al. (2019) Gut microbiome-gut dysbiosis-arterial hypertension: New horizons. Current Hypertension Reviews 15(1): 40-46.

19. Zhu TH, Zhu TR, Tran KA, Sivamani RK, Shi VY (2018) Epithelial barrier dysfunctions in atopic dermatitis: A skin-gut-lung model linking microbiome alteration and immune dysregulation. Brit J Dermatol 179(3): 570-581.

$\begin{array}{ll}\text { BIOMEDICAL } & \text { Assets of Publishing with us } \\ \text { RESEARCHES } & \text { - Global archiving of articles } \\ & \text { - Immediate, unrestricted online access } \\ & \text { - Rigorous Peer Review Process } \\ \end{array}$

